\title{
Fine tuning of the default depth and rate of ablation of the epithelium in customized trans-epithelial one-step superficial refractive excimer laser ablation
}

\author{
Michael Goggin ${ }^{1 *} \mathbb{D}$, Peter Stewart ${ }^{2}$, Vikija Andersons ${ }^{3}$ and Giuseppe Criscenti ${ }^{4}$
}

\begin{abstract}
Purpose: To fine tune the default depth and rate of ablation of the epithelium in $\mathrm{cTen}^{\mathrm{TM}}$ customized trans-epithelial one-step superficial refractive surgery by the comparison between the attempted post-operative ideal corneal shape and the achieved corneal shape.

Methods: 88 consecutive eyes in 64 patients undergoing trans-epithelial superficial excimer ablation using the iVis laser Suite for either myopic/astigmatic or hyperopic/astigmatic refractive error. Each patient had at least 3 months of post-operative follow-up. Topographic examination of all eyes was carried out pre-operatively and at least 3 months post-operatively using the Precisio ${ }^{\mathrm{TM}}$ surgical topographer. The comparison of these two measurements yielded values for depth, volumes and rates of ablated corneal tissue. By determining the different ablation rates of stroma and epithelium, a refinement of the depth of epithelium to be removed and a refinement of the stromal ablation were calculated.

The mathematical model was applied on each one of the 88 clinical cases and the parameters for the fine tuning of the default depth and rate of ablation of the epithelium were determined using the least squares method.

Results: The calculated pure stromal ablation rate was less than the average epithelium/stroma ablation rate used in planning the treatments by a factor of 0.96 . The epithelial thickness predefined ablation assumption used to plan removal of the epithelium was adjusted considering the measured ablation and a radial adjustment function established for the fine tuning of the laser radial efficiency and allowing for the normal thickening of the epithelium in the peripheral cornea. From a clinical point of view, this methodology produces an improvement of the efficacy and a reduction of the variance of the clinical results.

Conclusion: Comparison of accurately measured pre and postoperative topographies yields accurately established ablation rates of stroma and epithelium in trans-epithelial one step superficial ablation.
\end{abstract}

Keywords: Transepithelial photorefractive keratectomy, Epithelial ablation, Epithelial thickness refinement, Stromal ablation rate refinement

\footnotetext{
* Correspondence: michael.goggin@sa.gov.au

'South Australian Institute of Ophthalmology, University of Adelaide,

Adelaide, Australia

Full list of author information is available at the end of the article
}

(c) The Author(s). 2019 Open Access This article is distributed under the terms of the Creative Commons Attribution 4.0 International License (http://creativecommons.org/licenses/by/4.0/), which permits unrestricted use, distribution, and reproduction in any medium, provided you give appropriate credit to the original author(s) and the source, provide a link to the Creative Commons license, and indicate if changes were made. The Creative Commons Public Domain Dedication waiver (http://creativecommons.org/publicdomain/zero/1.0/) applies to the data made available in this article, unless otherwise stated. 


\section{Background}

Photorefractive keratectomy (PRK) is commonly performed in conjunction with manual removal of the central 6 to $9 \mathrm{~mm}$ of corneal epithelium by one of several methods. These include mechanical debridement with a spatula or similar instrument, an automated brush or with a keratome, sometimes facilitated by prior exposure of the epithelium to alcohol. The area thus denuded of epithelium is of necessity larger than the area of stromal ablation and invariably has an irregular edge [1-3].

With the manual removal of the epithelium, significant differences in UDVA, pain score, level of haze and complete epithelial healing time in the early postoperative period were detected by comparison with the laser removal approach [3].

In the last couple of decades, the possibility to remove automatically the corneal epithelium in a single-step procedure using a laser source represented a new alternative for laser refractive error correction [4].

The iRes excimer laser (iVisTechnologies, Taranto, Italy) performs PRK by ablating the epithelium to a pre-set default constant depth and ablating the customized stromal depth, all in a single surgical step. This technique specifically restricts the epithelial removal exactly to fit the chosen area of stromal ablation and provides a regular curvilinear post-operative epithelial edge [5-10].

However, to achieve a successful epithelial ablation, an assumption of epithelial thickness based on the manufacturer's laboratory measurements is made. A proprietary pre-set epithelial thickness is used for this technique which assumes thinner epithelium centrally than peripherally. However, the use of a default pre-set epithelial depth might induce an error in stromal ablation if the assumption is not correct.

The first aim of this study is to establish whether the default pre-set epithelial depth assumption is correct by comparison of the attempted ablations with that achieved, using a topographic measure of the ablation of both stroma and epithelium combined. The second aim of this study is to establish if the assumed epithelium and stroma average tissue ablation rate is correct.

\section{Methods}

The diagnostic analysis and the surgical treatments described in this study were performed with iVis Suite platform (iVis Technologies, Taranto, Italy) which include the surgical topographer Precisio ${ }^{\mathrm{mm}}$, the dynamic pupilometer pMetrics $^{\mathrm{m}}$, the Corneal Interactive Programmed Topographic Ablation CIPTA ${ }^{\circ}$ software, the 1 $\mathrm{kHz}$ excimer laser IRES ${ }^{\mathrm{Tw}}$ and the iVerify ${ }^{\mathrm{Tm}}$ statistical web application. Precisio ${ }^{\text {tw }}$ is a surgical topographer, designed for customized refractive surgery, equipped with a dedicated eye-tracker system that can detect the anterior corneal shape with a repeatability lower than $3 \mu \mathrm{m}$. The software medical device CIPTA $^{\circ}$ is designed to plan the customized tissue volume to be ablated defining the ideal corneal shape needed to achieve the desired correction and consequently calculate the volume of ablation as the difference between the Precisio ${ }^{\mathrm{mi}}$ detected anterior corneal shape and the ideal corneal shape.

In this study, 88 normal eyes in 64 patients underwent one-step customized trans-epithelial ablations using the iRES excimer laser. The surgeries were performed in two private refractive surgery centers in Australia (Brisbane Laser Sight Clinic, Brisbane, and Ashford Advanced Eye Care, Adelaide).

The patients included in this study met the following inclusion criteria:

- Over the age of 18

- With a refractive defect of: - Myopia

- Hyperopia

- Simple and compound astigmatism

- Mixed astigmatism

- Planned and executed customized trans-epithelial one step superficial keratorefractive surgery cTen ${ }^{\text {TM }}$ with a default tissue ablation rate and a default constant preset epithelial thickness, thinner centrally than peripherally

- Having a repeatable Precisio ${ }^{\text {ta }}$ topographer exam acquired either for the pre-operative planning exam, as well as for the 3 months follow-up post-operative exam, with valid maps covering a corneal diameter of $8 \mathrm{~mm}$ or greater

The repeatability analysis of the acquired exam is automatically performed by the Precisio ${ }^{\mathrm{Tm}}$ surgical topographer. It compares the first topographic examination with the second one that is taken immediately after the first as a routine procedure. The difference between the first and second topographic measurements on each mapped location is accepted only if it is $3 \mu \mathrm{m}$ or less throughout a minimum $6 \mathrm{~mm}$ central corneal zone. In addition, with iris and pupil registration at each step with this device (the pre-operative examination, the surgery and the post-operative examination), the $\mathrm{X}, \mathrm{Y}$ and rotational location of topographic change from the ablation can be established.

The achieved ablations are measured as the difference between the anterior pre-operative corneal shape and the anterior post-operative shape which are both detected using the Precisio ${ }^{\mathrm{Tw}}$ surgical topographer.

The attempted anterior post-operative shapes are determined as the difference between the anterior preoperative corneal shapes detected by the Precisio ${ }^{\text {th }}$ 
topographer and the executed customized ablation profiles planned with CIPTA ${ }^{\text {tw }}$ software.

The comparison between the attempted ablation and the achieved ablation is executed, on a point by point basis, in a radial direction, by means of the iVerify $^{\text {tw }}$ statistical application which allows the determination of the effective radial ablation rate analyzing the point-by-point local difference between the attempted ablation and the achieved ablation over a large database.

Surgeries were performed using the $\mathrm{iRES}^{\mathrm{m}}$ excimer laser which ablates at a tunable repetition rate up to $1000 \mathrm{~Hz}$ but adjusted to a constant frequency beam delivery on the cornea equal to $5 \mathrm{~Hz} / \mathrm{mm}^{2}$ to avoid thermal effects and achieve smooth profiles. During the whole treatment, the laser spot is delivered with a constant fluence of energy per unit of time.

The trans-epithelial approach described above was delivered in one step with a customized stromal ablation pattern based on the individual patient's refractive error and tailored to their topographic pattern. The customized area and outline of the epithelial ablation correspond to the area and outline of the stromal refractive ablation. Considering this approach, not all ablations are circular and all are "customized" so the effect of the ablation on the eye to be treated is calculated in order to have optimal edge profiles.

The post-operatively established changes in corneal shape were compared with the predicted ablation depths. The comparison was done in the center of the ablation as well as at varying radial distances (designated " $j$ ") from the center of the ablation (for mapping purposes designated as location "0;0") up to the radius of $5 \mathrm{~mm}$ from the center, where $\mathrm{j}=$ radial distances of $0.5 \mathrm{~mm}$, $1.0 \mathrm{~mm}, 1.5 \mathrm{~mm}, 2.0 \mathrm{~mm}, 2.5 \mathrm{~mm}, 3.0 \mathrm{~mm}, 3.5 \mathrm{~mm}$, $4.0 \mathrm{~mm}, 4.5 \mathrm{~mm}$ and $5.0 \mathrm{~mm}$. These data were collected on a Cartesian grid of $100 \mu \mathrm{m}$ at each one of the following annuli; $0.5 \mathrm{~mm}+/-100 \mu \mathrm{m}, 1.0 \mathrm{~mm}+/-100 \mu \mathrm{m}, 1.5 \mathrm{~mm}+$ / $-100 \mu \mathrm{m}, 2.0 \mathrm{~mm}+/-100 \mu \mathrm{m}, 2.5 \mathrm{~mm}+/-100 \mu \mathrm{m}, 3.0 \mathrm{~mm}+/$ $-100 \mu \mathrm{m}, 3.5 \mathrm{~mm}+/-100 \mu \mathrm{m}, 4.0 \mathrm{~mm}+/-100 \mu \mathrm{m}, 4.5 \mathrm{~mm}+/$ $-100 \mu \mathrm{m}$ and $5.0 \mathrm{~mm}+/-100 \mu \mathrm{m}$. This covers a possible ablated zone of up to $10 \mathrm{~mm}$. Mean values within these areas were determined and compared (attempted versus achieved).

The best approximation of the achieved ablation depth thus derived at the center of the ablation $(0 ; 0)$ was compared with the attempted ablation calculated in the center of the ablation $(0 ; 0)$, according to the following function:

$$
\mathrm{R}_{\mathrm{i}}=\mathrm{xEs}_{\mathrm{i}}+(\mathrm{y}-1) \mathrm{Ep} \text {, }
$$

where

$\mathrm{i}=1 \ldots \mathrm{n}, \mathrm{n}=$ treated eye number;

$R_{i}$ is the achieved value of ablation depth including the epithelium for each treated eye;
$\mathrm{Es}_{\mathrm{i}}$ is the expected value of the ablation depth of the stroma for each treated eye;

Ep is the default epithelial depth value used to ablate the epithelium;

$\mathrm{x}$ is the correction constant to be defined for the stromal ablation;

$y$ is the correction constant to be defined for the epithelium ablation;

(y-1)Ep is the corrected constant of the total epithelium ablation depth;

$\mathrm{x}$ and $(\mathrm{y}-1)$ Ep are calculated applying the least squares method.

The determination of the coefficients of the above function provided the best corrective $\mathrm{x}$ constant for the stromal ablation rate. The proprietary IRES laser energy factor used in the treatments (which is an average of stromal and epithelial ablation rates) was multiplied by this constant to define the new pure stromal ablation rate. The best corrected (y-1)Ep constant of the ablation depth for the epithelium was also similarly established and applied to the default constant value at the center of the ablation $(0 ; 0)$.

The determination of the $(\mathrm{y}-1) \mathrm{Ep}_{\mathrm{j}}$ values at $\mathrm{j}$ radial distance from the center of the ablation $(0 ; 0)$ up to the radius of $5 \mathrm{~mm}$ from the center (the $10 \mathrm{~mm}$ maximum ablation zone of the device) was obtained by the best approximation of the mean achieved ablation depth versus the expect ablation depth calculated according to the following function:

$$
R_{i}=x \mathrm{Es}_{\mathrm{ij}}+(\mathrm{y}-1) \mathrm{Epi}_{\mathrm{j}} \text {. }
$$

where

$\mathrm{i}=1 \ldots \mathrm{n}, \mathrm{n}=$ treated eye number;

$\mathrm{j}=$ radial distance $(0.5 \mathrm{~mm}, 1 \mathrm{~mm}, 1.5 \mathrm{~mm}, 2 \mathrm{~mm}$, $2.5 \mathrm{~mm}, 3 \mathrm{~mm}, 3.5 \mathrm{~mm}, 4 \mathrm{~mm}, 4.5 \mathrm{~mm}$ and $5 \mathrm{~mm}$ );

$\mathrm{R}_{\mathrm{i}}$ is the mean achieved value of ablation depth including the epithelium for each treated eye at $j$ distance from the center of the ablation;

$\mathrm{Es}_{\mathrm{i}}$ is the expected value of the stromal ablation depth for each treated eye at $j$ distance from the center of the ablation;

$\mathrm{x}$ is the correction constant as defined for the stromal ablation above;

$(y-1) E p_{j}$ is the corrected constant of total epithelium ablation depth defined for each $j$ radial distance from the center of the ablation $(0 ; 0)$.

The determination of the $(\mathrm{y}-1) \mathrm{Ep}_{\mathrm{j}}$ coefficients provided the data set for each $\mathrm{j}$ radial distance from $0.5 \mathrm{~mm}$ radius up to the radius of $5 \mathrm{~mm}$ from the center of the ablation $(0 ; 0)$, again covering the maximum ablation zone of 10 $\mathrm{mm}$.

Finally, the function that describes the epithelial thickness used to ablate the epithelium in one step superficial refractive surgery, defined as corrected $\mathrm{f}$ (radial distance), is obtained by fitting of the $(y-1) \mathrm{Ep}_{j}$ values with a 
proprietary second order function related to the distance from the center of the ablation $(0 ; 0)$ up to $5 \mathrm{~mm}$. This approach allows the gradual radial thickening of the epithelium in normal corneas.

\section{Statistical analysis}

The measure of the Least Squares approximation goodness was assessed by the determination coefficient $\left(R^{2} \geq 50 \%\right)$ and the correlation coefficient of Bravais-Pearson $(R>70 \%)$. The $\mathrm{R}$ correlation coefficient was calculated to validate the hypothesis of a linear interdependence between the real ablation data set and the attempted ablation data set through the $x$ and $(y-1)$ Ep coefficients. It ranges in value from -1 to +1 , indicating perfect negative correlation at -1 , absence of correlation at zero, and perfect positive correlation at +1 . The coefficient of correlation $\mathrm{R}$ should be greater than 0.70 to show a good linear correlation. The coefficient of determination, denoted by $\mathrm{R}$ [2], was calculated to measure the percentage of variability of $R$ in function of the variability of E. It ranges in value from 0 to $100 \%$. The coefficient of determination $\mathrm{R}^{2}$ should be greater than or equal to $50 \%$.

After the determination of the $\mathrm{x}$ and (y-1)Epij values, the weighted mean ratios of Ri values vs. (xEsii $+(y-1)$ Epij) values are calculated at each radial distance $j$ from the center of the ablation up to a distance of $3 \mathrm{~mm}$ from the center. The percent mean error $\mathrm{E} \%$ and the precision $\mathrm{D}$, expressed as follows, should meet the following values for each $\mathrm{x}$ and ( $\mathrm{y}-1)$ Epij:

$$
E \%=\left(\text { Weighted mean }\left(\frac{R_{i}}{\left(x E s_{i}+(y-1) E p_{i j}\right)}-1\right)\right) * 100 \leq 30 \%
$$

$\mathrm{D} \leq 5 \%$.

To assess the reliability of the obtained results, according to the Gaussian assumption, the $95 \%$ confidence interval (CI) for the mean $M$ of the ratios between observed data and estimated data was considered at all radial distances and the corresponding precision $D$ was calculated and analyzed:
$C I=M \pm k^{*} S E$.

$D=k^{*} S E^{*} 100 \leq 5 \%$,

where $k=1.96$ is the $\alpha / 2$ quantile of the standard normal distribution with $\alpha=0.05$ and $S E$ is the standard error equal to the standard deviation divided by the square root of the treated eye number: $\mathrm{SD} / \sqrt{\mathrm{n}}$.

\section{Results}

This study evaluated 88 eyes of 64 patients (51 right eyes and 37 left eyes). Demographic information of the population are summarized in Table 1.

The mean follow-up time was $3.96 \pm 1.15$ months (range: 3 to 7 months). The mean spherical equivalent was $-2.08 \pm 2.17 \mathrm{D}$ (range: -7.30 to $2.58 \mathrm{D}$ ). Approximation with the Least Squares method of the achieved ablation depth determined at each distance "j" from the center of the ablation $(0 ; 0)$ vs. the expected ablation calculated at each distance " $j$ " from the center of the ablation $(0 ; 0)$, for each one of the 88 following functions is as follows:

$$
\begin{aligned}
& R_{i j}=x E s_{i j}+y E p i j \text { where } i=1 \ldots n, n=88 j=0,0.5,1 \\
& \ldots .5 \text {; }
\end{aligned}
$$

The following results were obtained (Table 2).

The correction constant $\mathrm{x}$ for pure stromal ablation versus the average ablation rate which includes both epithelium and stroma is equal to 0.96 . This indicates that the previous default value was under-ablating the corneal stroma by approximately $4.0 \%$. It can be observed that the correlation coefficient $\mathrm{R}$ is greater than $70 \%$, so there is a linear dependence between real and attempted ablation depths. Moreover, the coefficient of determination $R^{2}$ is greater than $50 \%$, thus the assumed model is correct. With respect to data reliability, the inequality $\mathrm{E} \% \leq 30 \%$ is satisfied as well as the inequality $\mathrm{D} \leq 5 \%$.

According to the results shown in the preceding section, the corrective constant $\mathrm{x}$ is set at 0.96 with the

\begin{tabular}{|c|c|c|c|c|c|c|c|}
\hline \multirow{2}{*}{\multicolumn{2}{|c|}{ Demographics }} & \multicolumn{4}{|l|}{ Sites } & \multirow{2}{*}{\multicolumn{2}{|c|}{ Site $1+$ Site 2}} \\
\hline & & \multicolumn{2}{|l|}{1} & \multicolumn{2}{|l|}{2} & & \\
\hline \multicolumn{2}{|c|}{ Number of eyes \& subjects } & \multicolumn{2}{|c|}{35 eyes of 24 enrolled subjects } & \multicolumn{2}{|c|}{53 eyes of 40 enrolled subjects } & \multicolumn{2}{|c|}{88 eyes of 64 enrolled subjects } \\
\hline & & $n$ & $\%$ & $n$ & $\%$ & $n$ & $\%$ \\
\hline \multirow[t]{2}{*}{ Gender } & Male & 7 & 29.2 & 12 & 30 & 19 & 29.7 \\
\hline & Female & 17 & 70.8 & 28 & 70 & 45 & 70.3 \\
\hline \multirow[t]{2}{*}{ Surgical eye } & Right & 20 & 57.1 & 31 & 58.5 & 51 & 58 \\
\hline & Left & 15 & 42.9 & 22 & 41.5 & 37 & 42 \\
\hline \multirow[t]{3}{*}{ Age (in years) } & Mean (SD) & \multicolumn{2}{|l|}{$37.9(13.4)$} & \multicolumn{2}{|c|}{$46.2(13.6)$} & \multicolumn{2}{|c|}{$42.8(14.2)$} \\
\hline & Minimum & \multicolumn{2}{|l|}{21.0} & \multicolumn{2}{|c|}{22.0} & \multicolumn{2}{|c|}{21.0} \\
\hline & Maximum & \multicolumn{2}{|l|}{68.0} & \multicolumn{2}{|c|}{70.0} & \multicolumn{2}{|c|}{70.0} \\
\hline
\end{tabular}
model described in the previous section to evaluate the

Table 1 Demographic information of the population used in this study 
Table 2 Obtained values for the corrective constants $x$ and $(\mathrm{y}-1) \mathrm{Ep}$, the parameters for the goodness of fit and the parameters for the data reliability assuming the model described in methods section

\begin{tabular}{lccccc}
\hline$x$ & $(y-1) E p$ & $R(\%)$ & $R^{2}(\%)$ & $E_{\%}(\%)$ & $D(\%)$ \\
\hline 0.96 & 10.459 & 96.08 & 92.31 & -0.58 & 3.63 \\
\hline
\end{tabular}

corrective constants (y-1)Epij where $\mathrm{j}$ is the radial distance from the center of the ablation and it ranges from $0 \mathrm{~mm}$ to $3 \mathrm{~mm}$ with a step of $0.5 \mathrm{~mm}$.

In Table 3, the values obtained for the corrective constants $(y-1)$ Epij and the corresponding reliability parameters are shown.

As shown in Fig. 1, the percentage radial thickening of the epithelium with respect to the default constant value ranged from -19.3 to $+12.1 \%$. In particular, a reduction of $19.3 \%$ of the default constant value was calculated in the central zone. At a radius of approximately $4 \mathrm{~mm}$, the default constant value was similar to the calculated one $(0.8 \%)$ while an increase of $12.1 \%$ of the constant default value was found to be necessary at the $5 \mathrm{~mm}$ radius annulus. These data were calculated depending on the effective solutions, using the least squares method, of the $R_{i j}=x s_{i j}+y E p i j$ equations up to $2.5 \mathrm{~mm}$ from the center, where the observed data were reliable according to the precision previously defined (less than $5 \%$ ). The data between 3.0 and $5.0 \mathrm{~mm}$, having a precision greater than $5 \%$, were extrapolated from the data.

\section{Discussion}

In this study, a method is described to fine tune the default depth and rate of ablation of the epithelium in $\mathrm{cTen}^{\mathrm{Tx}}$ customized trans-epithelial one-step superficial refractive surgery by comparing the attempted postoperative ideal corneal shape and the achieved corneal shape. The cTen surgical approach has been demonstrated to have high stability at 1 month postoperative follow up [11]. Each patient had at least 3 months of post-operative follow-up and the mean follow-up time was $3.96 \pm 1.15$ months (range: 3 to 7 months).

The calculated pure stromal ablation rate was less than the average epithelium/stroma ablation rate used in planning the treatments by a factor of 0.96 . The epithelial thickness assumption used to plan removal of the epithelium was adjusted on the basis of the measured ablation

Table 3 Corrective constants (y-1)Epij values and corresponding reliability parameters

\begin{tabular}{llllllll}
\hline & \multicolumn{6}{c}{ Radial Distance from the center of the ablation (in $\mathrm{mm}$ ) } \\
\cline { 2 - 8 } & 0 & 0.5 & 1 & 1.5 & 2 & 2.5 & 3 \\
\hline (y-1)Epij & 10.361 & 10.100 & 9.293 & 8.036 & 6.549 & 5.481 & 5.296 \\
$E_{\%}(\%)$ & -0.62 & -0.66 & -0.55 & -0.25 & 0.29 & 1.16 & 3.16 \\
$D(\%)$ & 3.63 & 3.67 & 3.83 & 4.09 & 4.43 & 4.69 & 5.67 \\
\hline
\end{tabular}

and a radial adjustment function established. This radial adjustment was calculated from the observed normal radial increase in epithelial thickness toward the periphery and was used to fine tune the laser's radial efficiency.

In order to perform trans-epithelial refractive laser ablation, the exact knowledge of the epithelial thickness and the ablation rate of the epithelium and of the stroma is mandatory. Excess ablation wastes stromal tissue and variations in epithelial thickness of the ablated area may influence refractive outcome $[1,2]$. Under-estimation of these parameters may contribute to a reduction of the optical zone while an incorrect evaluation of the epithelium profile to be ablated may affect the aspherical treatment outcome due to the different ablation rate and, consequently, different ablation profile. Under- or overestimation of the rate of ablation either of the epithelium or stromal tissue will also influence the refractive outcome.

The method of defining the customized ideal shape needed to optimize the quality of vision, instead of the most common approach of printing a refractive lens onto the cornea, allows for the determination of the effectively achieved ablation volume, inclusive of postoperative re-epithelization process and induced biomechanical changes. In fact, the differences between the attempted and the achieved volume of ablation may be effectively determined by means of the comparison between the attempted ideal corneal shape and the achieved post-operative corneal shape. By comparison of the attempted vs. achieved ablation patterns over a large database, using accurate topographic information, the assumed values of these parameters can be refined. Prior to refinement, the epithelial thickness was assumed to have a proprietary default profile across the whole ablated area (thinner centrally than peripherally) and the stromal ablation rate equal to the epithelial ablation rate. Examination of the true stromal ablation rate and true epithelial thickness suggests a refined pure stromal ablation rate increment of $4.0 \%$ on the rate used for the treatments in the study. A radial variation of the epithelial thickness to be ablated is suggested with a reduction of the assumed epithelial thickness equal to $19.3 \%$ in the center of the ablated area and an increase of the assumed epithelial thickness equal to $12.1 \%$ at the $5 \mathrm{~mm}$ radius (the maximum area that can be ablated with the iRES excimer laser). It should be pointed out that the apparent epithelial thickness established here is in terms of ablation rates. The used method includes the effect of the radial efficiency of the laser and the effect on corneal thickness of corneal epithelial regrowth and healing which may not return to an epithelium that is exactly the same as prior to surgery. Consequently, it may be different to epithelial thickness measured by optical systems but is a measure of "functional" epithelial thickness. 


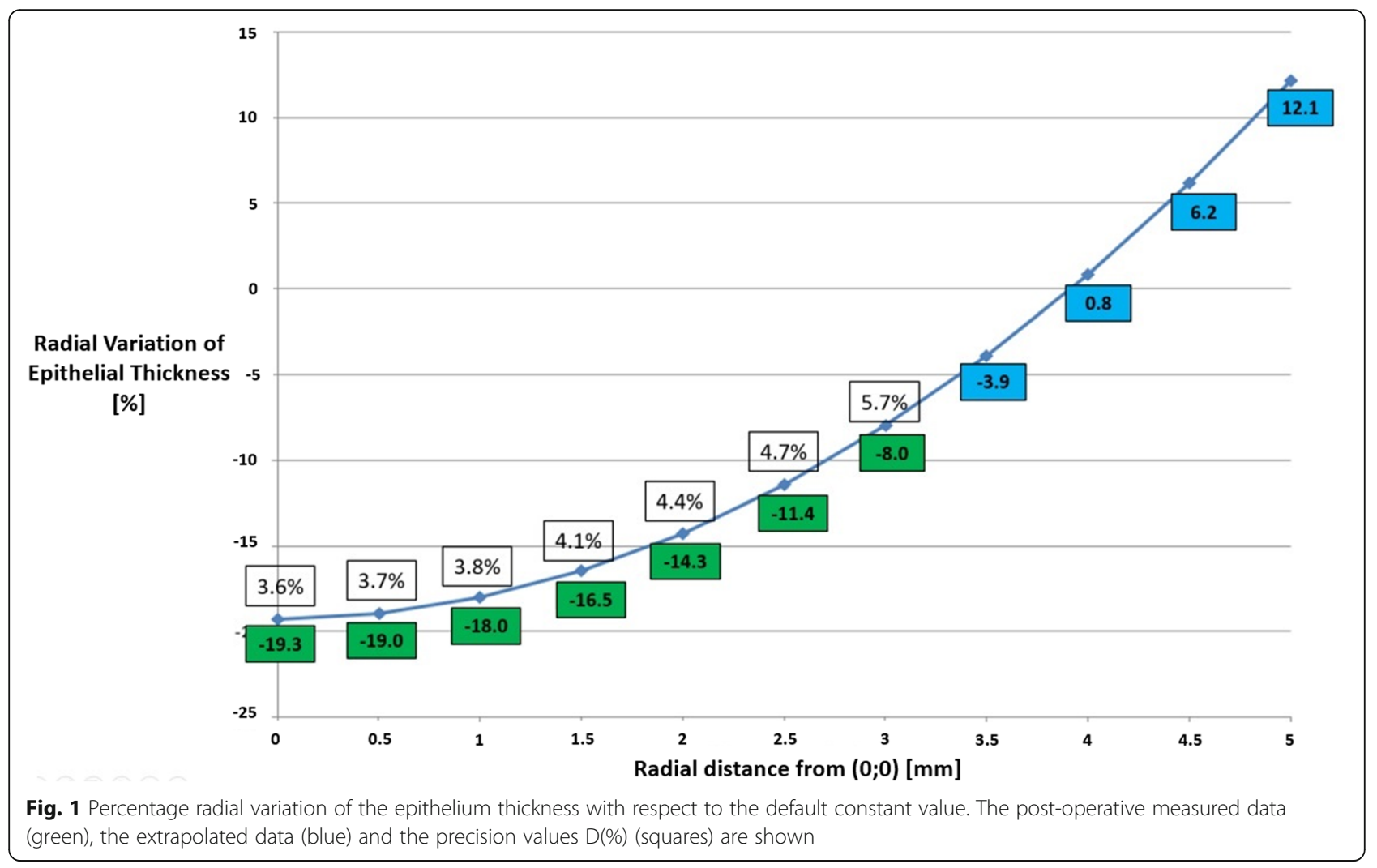

In common with all other laser systems, an ablation rate of corneal tissue is established by the manufacturer's laboratory measurements and applied in treatment planning. This proprietary average includes ablation of both stroma and epithelial tissue. Comparison of attempted ablations versus achieved ones, by topographic measures, can be also used to refine this value and to refine the value assumed for epithelial thickness.

Trans-epithelial one step refractive excimer laser ablation $\mathrm{cTen}^{\mathrm{Tu}}$ is comparable in terms of outcomes to traditional alcohol-assisted or manual removal of epithelium [12-15]. Further, there is some evidence showing that it may provide better visual outcome in eyes with low to moderate myopia when compared with LASIK, LASEK and manual epithelial removal PRK [16]. With the very high ablation rate of the IRES excimer laser $(1000 \mathrm{~Hz})$, this procedure is performed rapidly as the excimer laser removes the epithelium and ablates the stroma in one single step [12]. There is less post-operative pain and faster epithelial healing probably because a precise zone of epithelium is removed only where the stromal ablation will take place $[14,17,18]$. Moreover, transepithelial single step superficial refractive surgery has significantly less post-operative corneal haze at 1,3 , 6 and 12 months when compared to conventional PRK [18].
Myopic and hyperopic ablations lead to opposite morphological changes and cause opposite postoperative epithelial and biomechanical changes. This is a possible drawback of our technique but accurate pre- and postoperative topographic information in conjunction with the Least Squares method, calculated for individual eyes, will allow one to limit this possible source of error without the need to study different ablation types.

Prior to this study, the iRES excimer laser ablated the epithelium to a default pre-defined depth (thinner centrally than peripherally). Refinement of the epithelial thickness assumption, both centrally and peripherally, allows more accurate ablation depth planning and the ablation of only the stromal tissue needed for refractive correction. In addition, an accurate estimation of stromal ablation rates allows for a better treatment plan and assists in achieving more accurate outcome, improvement of the efficacy and reduction of the variance of the clinical results.

Ongoing audit of these data and precise epithelial mapping will further contribute to better outcome in the future.

\section{Conclusion}

The comparison of accurately measured pre- and postoperative topographies yields accurately established ablation rates of stroma and epithelium in trans-epithelial 
one step superficial ablation. Modification of the assumed rates to the rates thus established may lead to more accurate topographic and consequently, more accurate refractive outcome with an improvement of the efficacy and a reduction of the variance of the clinical results. The analytical techniques used in this study are applicable to further data examining ablation rates as they may vary with patient age or disease state or with different ablation techniques.

\section{Acknowledgements}

The data described in this article are archived by Associate Professor Michael Goggin, Ashford Advanced Eye Care, Adelaide, Australia and by iVis Technologies, Taranto, Italy.

\section{Authors' contributions}

MG: Patient data collection and analysis, preparation, review and major contribution of the manuscript. PS: Patient data collection and review of the manuscript. VA: Patient data collection and review of the manuscript. GC: Preparation and review of the manuscript. All authors read and approved the final manuscript.

\section{Funding}

Ethics submission financed by iVis Technologies. No other funding required.

\section{Availability of data and materials}

Not applicable.

\section{Ethics approval and consent to participate}

Ethics committee approval was obtained from Bellberry Ltd. Human Research Ethics Committee (Adelaide, Australia). The study adhered to the tenets of the Helsinki Declaration.

\section{Competing interests}

Data analysis support was provided by iVis Technologies, Taranto, Italy. Michael Goggin, Peter Stewart and Vikija Andersons report no financial interest in any of the products of the study.

\section{Author details}

'South Australian Institute of Ophthalmology, University of Adelaide, Adelaide, Australia. ${ }^{2}$ Brisbane Laser Sight Clinic, Brisbane, Australia. ${ }^{3}$ Ashford Advanced Eye Care, Adelaide, Australia. ${ }^{4}$ iVis Technologies, Taranto, Italy.

Received: 11 April 2019 Accepted: 14 October 2019

Published online: 03 December 2019

\section{References}

1. Tomás-Juan J, Murueta-Goyena Larrañaga A, Hanneken L. Corneal regeneration after photorefractive keratectomy: a review. J Optom. 2015;8(3):149-69.

2. Shapira Y, Mimouni M, Levartovsky S, Varssano D, Sela T, Munzer G, et al. Comparison of three epithelial removal techniques in PRK: mechanical, alcohol-assisted, and transepithelial laser. J Refract Surg. 2015;31(11):760-6.

3. Bakhsh AM, Elwan SAM, Chaudhry AA, El-Atris TM, Al-Howish TM. Comparison between transepithelial photorefractive keratectomy versus alcohol-assisted photorefractive keratectomy in correction of myopia and myopic astigmatism. J Ophthalmol. 2018;2018:5376235.

4. Kaluzny BJ, Cieslinska I, Mosquera SA, Verma S. Single-step transepithelial PRK vs alcohol-assisted PRK in myopia and compound myopic astigmatism correction. Medicine (Baltimore). 2016;95(6):e1993.

5. Kapadia MS, Wilson SE. Transepithelial photorefractive keratectomy for treatment of thin flaps or caps after complicated laser in situ keratomileusis. Am J Ophthalmol. 1998:126(6):827-9.

6. Chen X, Stojanovic A, Zhou W, Utheim TP, Stojanovic F, Wang Q. Transepithelial, topography-guided ablation in the treatment of visual disturbances in LASIK flap or interface complications. J Refract Surg 2012; 28(2):120-126.

7. Chen X, Stojanovic A, Nitter TA. Topography-guided transepithelial surface ablation in treatment of recurrent epithelial ingrowths. J Refract Surg. 2010; 26(7):529-32.
8. Giessler S, Duncker Gl. Recurrent corneal erosion after mechanical trauma. Results of transepithelial phototherapeutic keratectomy. Ophthalmology. 2001;98(10):950-4

9. Holzer MP, Auffarth GU, Specht H, Kruse FE. Combination of transepithelial phototherapeutic keratectomy and autologous serum eyedrops for treatment of recurrent corneal erosions. J Cataract Refract Surg. 2005;31(8):1603-6.

10. Stojanovic A, Zhang J, Chen X, Nitter TA, Chen S, Wang Q. Topography-guided transepithelial surface ablation followed by corneal collagen cross-linking performed in a single combined procedure for the treatment of keratoconus and pellucid marginal degeneration. J Refract Surg. 2010;26(2):145-52.

11. Zhou W, Reinstein DZ, Chen X, Chen S, Xu Y, Utheim TP, et al. Transepithelial topography-guided ablation assisted by epithelial thickness mapping for treatment of regression after myopic refractive surgery. J Refract Surg. 2019;35(8):525-33.

12. Luger MH, Ewering T, Arba-Mosquera S. Consecutive myopia correction with transepithelial versus alcohol-assisted photorefractive keratectomy in contralateral eyes: one-year results. J Cataract Refract Surg. 2012;38(8):1414-23.

13. Aslanides IM, Padroni S, Arba Mosquera S, loannides A, Mukherjee A. Comparison of single-step reverse transepithelial all-surface laser ablation (ASLA) to alcohol-assisted photorefractive keratectomy. Clin Ophthalmol. 2012;6:973-80.

14. Wang DM, Du Y, Chen GS, Tang LS, He JF. Transepithelial photorefractive keratectomy mode using SCHWIND-ESIRIS excimer laser: initial clinical results. Int J Ophthalmol. 2012;5(3):334-7.

15. Thomann UM, Schipper I. Customized transepithelial no-touch ablation as therapeutic option. J Cataract Refract Surg. 2010;36(7):1244-5.

16. Ghadhfan F, Al-Rajhi A, Wagoner MD. Laser in situ keratomileusis versus surface ablation: visual outcomes and complications. J Cataract Refract Surg. 2007:33(12):2041-8.

17. Buzzonetti L, Petrocelli G, Laborante A, Mazzilli E, Gaspari M, Valente P, et al. A new transepithelial phototherapeutic keratectomy mode using the NIDEK CXIII excimer laser. J Refract Surg. 2009;25(Suppl 1):S122-4.

18. Kanitkar KD, Camp J, Humble H, Shen DJ, Wang MX. Pain after epithelial removal by ethanol-assisted mechanical versus transepithelial excimer laser debridement. J Refract Surg. 2000;16(5):519-22.

\section{Ready to submit your research? Choose BMC and benefit from:}

- fast, convenient online submission

- thorough peer review by experienced researchers in your field

- rapid publication on acceptance

- support for research data, including large and complex data types

- gold Open Access which fosters wider collaboration and increased citations

- maximum visibility for your research: over $100 \mathrm{M}$ website views per year

At $\mathrm{BMC}$, research is always in progress.

Learn more biomedcentral.com/submissions 